\title{
Effect of Crystal Orientation on Imaging Contrast and Sputter Results during Focused Ion Beam Milling of Cu studied by FIB, EBSD, SEM, and AFM
}

\author{
U. Wendt*, G. Nolze**, H. Heyse* \\ * Institute of Materials and Joining Technology, Otto-von-Guericke University, PF 4120, 39016 \\ Magdeburg, Germany \\ ** Federal Institute for Materials Research and Testing (BAM), Unter den Eichen 87, \\ 12205 Berlin, Germany
}

During focused ion beam microscopy (FIB) of crystalline materials imaging contrast and milling result are effected by orientation of the crystals with respect to the incident ion beam. This is due to the possibility of ion channeling along preferred crystal directions which effects the depth at which interaction between ions and specimen atoms takes place [1]. As a result of channeling emission of ion induced secondary electrons (iiSE) and secondary ions (SI) as well as the sputter rate decreases. Theoretical channeling orientations and critical angles can be calculated [2].

These effects have been studied quantitatively for polycrystalline recrystallized $\mathrm{Cu}$ as a typical model case.

Crystal orientation map was obtained by electron backscatter diffraction (EBSD) of a polished specimen before ion milling. Selected areas were FIB milled using a beam dose between 0.5 and 2 $\mathrm{nC} / \mu \mathrm{m}^{2}$ (Fig. 1). Grains with high iiSE emission (f.e. grain "b" in Fig. 2) had orientations (Fig. 3) which show no or only little channeling effect.

Topography after FIB sputtering depends on grain orientation (Fig. 4). Some grains were almost featureless whereas others had a rippled or dotted topography. Sputter depth, as measured by atomic force microscopy (AFM) (Fig. 5), changes linearely with increasing gray value of the grains in the FIB iiSE image (Fig. 6). Topography of the sputtered surfaces was quantified using normalized surface area $R_{S}=A / A_{o}$ (with $A=$ true surface area and $A_{o}=$ projected area) [3] as a topometry parameter. $R_{S}$ correlates with orientation of the grains.

To study the influence of sputter time on topography, a grain with an almost [011] orientation marked "g" in Fig. 2 was choosen. Four squares were milled within this grain using a beam dose between 0,5 and $2 \mathrm{nC} / \mu \mathrm{m}^{2}$ (Fig. 7). Sputter depth increases linearly with time (Fig. 8). The ripple like topography as shown by AFM images (Fig. 9) maintaines its principal structure even at a sputter depth of more than $3 \mu \mathrm{m}$. The peak to peak distance varies between 150 and $310 \mathrm{~nm}$. The depth of the ripples was $50 \mathrm{~nm}$ at a milling dose of $0.5 \mathrm{nC} / \mu \mathrm{m}^{2}$ (ion beam current $373 \mathrm{pA}$ ) and keeps constant at a value of about $80 \mathrm{~nm}$ during further milling.

The results obtained on $\mathrm{Cu}$ are useful for the manufacturing of MEMS devices and for the generation of surfaces with a specific topography. These surfaces are interesting $\mathrm{f}$. e. with regard to adsorption, optical behaviour, and wear properties.

\section{References}

[1] Y. Yahiro et al., J. Electron Microsc., 53 (2004) 571.

[2] B.W. Kempshall et al., J.Vac.Sci.Technol., B 19 (2001) 749.

[3] E.E. Underwood, Acta stereol., 10 (1991) 149. 


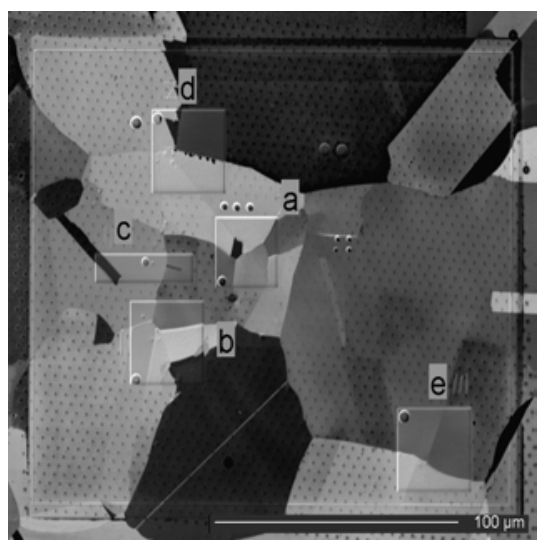

Fig. 1. FIB image (iiSE) of a $\mathrm{Cu}$ specimen with four FIB milled regions and markers.

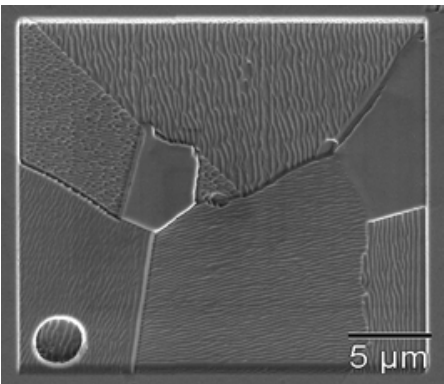

Fig. 4. SEM image (SE) of the area in Fig. 2 after FIB milling with a mill dose of $0,53 \mathrm{nC} / \mu \mathrm{m}^{2} .2$ and 4 after FIB sputtering.

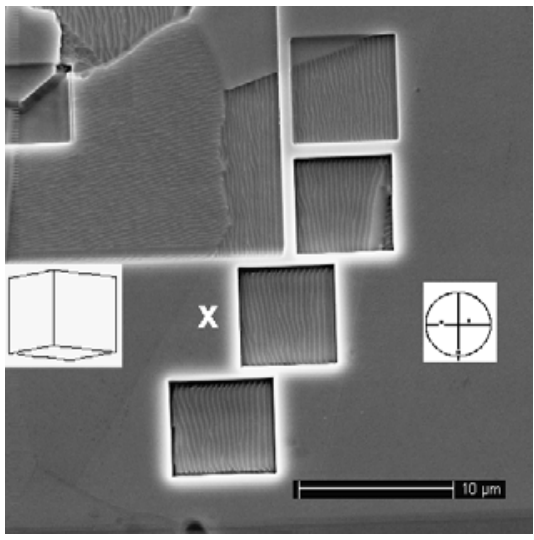

Fig. 7. SEM image with overlayed orientation and pole figure of grain "g" in Fig. 2 after FIB milling for different times.
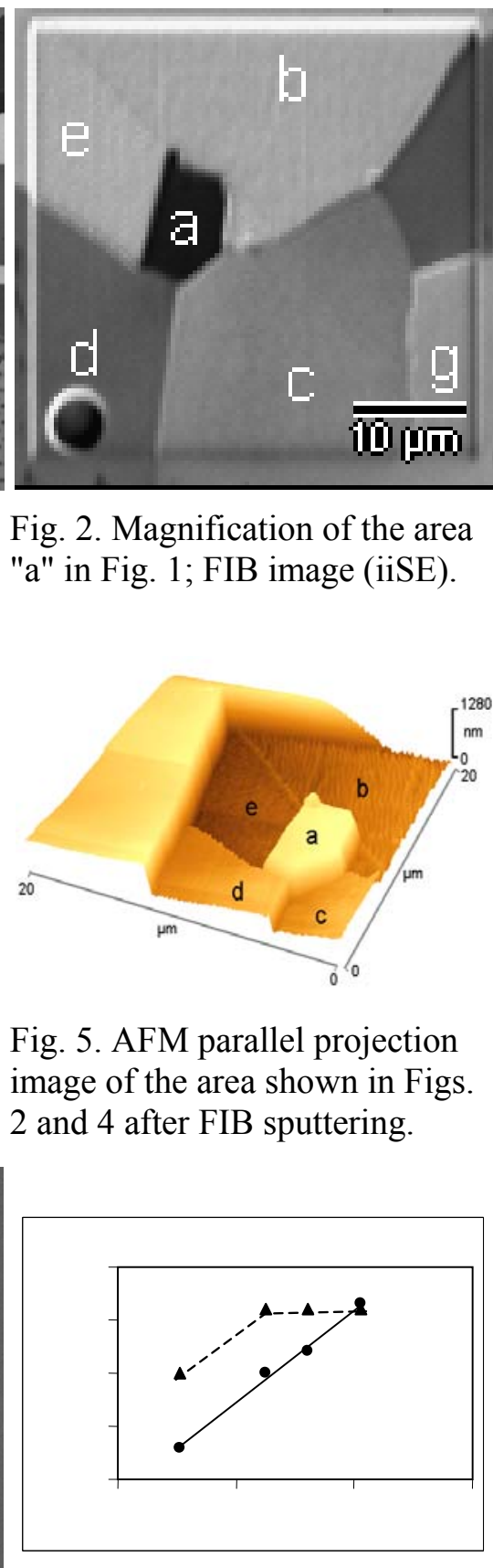

Fig. 2. Magnification of the area "a" in Fig. 1; FIB image (iiSE).

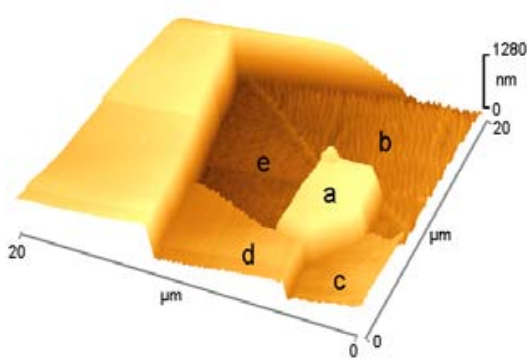

Fig. 5. AFM parallel projection image of the area shown in Figs.

Fig. 8. FIB milling dose (md) $\left[\mathrm{nC} / \mu \mathrm{m}^{2}\right]$ vs. sputter depth (d) for the four squares in Fig. 7 and depth of the ripples (r).

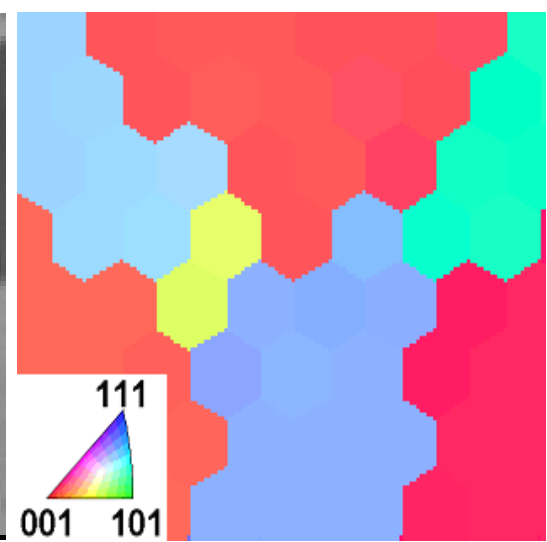

Fig. 3. EBSD orientation map as inverse pole figure of the region in Fig. 2.

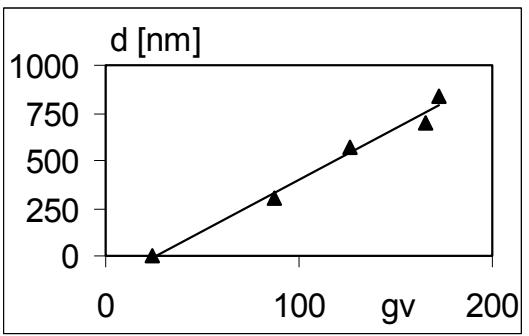

Fig. 6. Gray value (gv) vs. sputter depth (d) of the grains in Figs. 2 and 5 .

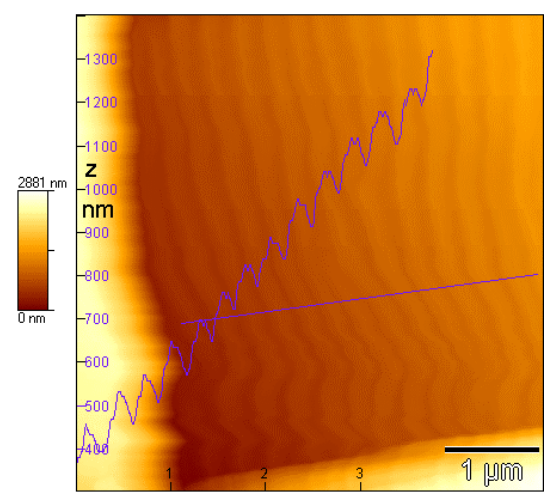

Fig. 9. AFM image of the square marked "x" in Fig. 7 and height profile along the marked line. 